\title{
Montgomery T-tube for the Management of Postintubation Tracheoesophageal Fistula with Synchronic Subglottic Laryngotracheal Stenosis
}

\author{
Zhigang $\mathrm{Li}^{1} \quad$ Chenxi Zhong ${ }^{1}$ \\ ${ }^{1}$ Department of Thoracic Surgery, Shanghai Chest Hospital, Shanghai, \\ Address for correspondence Zhigang Li, MD, PhD, Department of \\ China \\ Thoracic Surgery, Shanghai Chest Hospital, 241 Huaihai West Road, \\ Shanghai 200433, China (e-mail: dr_lizhigang@163.com). \\ Thorac Cardiovasc Surg Rep 2014;3:61-63.
}

\begin{abstract}
Keywords

- thoracic surgery

- airway

- larynx

A postintubation tracheoesophageal fistula (TEF) complicated with subglottic laryngotracheal stenosis (SLTS) is a challenge to surgical repair. Laryngotracheal resection and primary reconstruction could result in treatment failure. In this report, we describe the successful management of two cases of postintubation TEF complicated with SLTS using a Montgomery T-tube because of the patients' contraindications for laryngotracheal resection and primary anastomosis.
\end{abstract}

\section{Introduction}

Airway stenosis and tracheoesophageal fistula (TEF) are the most common complications of endotracheal intubation or tracheostomy. Tracheal stenosis complicated with TEF at the cuff area is common and is usually treated by a segmental tracheal resection and primary anastomosis with esophageal closure. ${ }^{1}$ Subglottic laryngotracheal stenosis (SLTS) occurs due to circumferential cricoid erosion by the endotracheal tube or proximal erosion of the cricoid by a high or violent tracheostomy, rather than by cuff compression-induced ischemia. Few studies have reported the clinical management of TEF complicated with SLTS, although laryngotracheal resection and primary reconstruction have been theoretically suggested. ${ }^{2,3}$

This report describes the successful management of two cases of postintubation TEF complicated with SLTS using a Montgomery T-tube because of the patients' contraindications for laryngotracheal resection and primary anastomosis.

\section{Case Reports}

\section{Case 1}

A 75-year-old man was admitted to our hospital, and TEF and SLTS were suspected because of an irritating cough after eating. The patient had been well until 3 months before, when he experienced a traffic accident causing a traumatic brain injury for which mechanical ventilation and tracheotomy were required. Bronchoscopy showed that the airway lumen at the cricoid level was completely occluded, and a giant TEF approximately $3.5 \mathrm{~cm}$ was observed below the occluded airway.

Because the patient had not fully recovered from the brain injury, a long-segment airway resection and reconstruction were contraindicated, and a Montgomery T-tube was selected as an alternative option.

After transtracheostomy intubation under general anesthesia, the surgery was performed through a low-collar incision to expose the thyroid and cricoid cartilages and the anterior wall of the involved trachea, which was then dissected bilaterally without any deliberate exposure of the recurrent laryngeal nerve. After reaching the fistula, a portion of the esophageal wall was harvested to facilitate closure on the tracheal side. The esophagus was closed using a two-layer technique, and then a left strap muscle flap was used to cover the repaired esophagus and interpose the airway. The tracheostomy stoma was expanded longitudinally. The intratracheal scar tissue was cleared. The cricoid cartilage was kept received

May 16, 2014

accepted after revision

June 20, 2014

published online

September 3, 2014
DOI http://dx.doi.org/

10.1055/s-0034-1386719. ISSN 2194-7635. (c) 2014 Georg Thieme Verlag KG
Stuttgart · New York

License terms

((1) $\circledast$ 
intact, but the submucosal hyperplastic tissue was removed. The subglottic and upper tracheal lumen was dredged, and the tracheal defect was repaired with continuous 4-0 polydioxanone suture (PDS; Ethicon, Somerville, New Jersey, United States) from outside the lumen. A Montgomery T-tube (15 mm in diameter; Boston Medical Product Inc., Westborough, Massachusetts, United States) supported the airway. A wedge plasty was made at the posterior wall of the proximal branch of the T-tube to facilitate the narrowness at cricoid. The upper edge of the T-tube was adjusted to $1 \mathrm{~cm}$ from the vocal cord with the distal end fixed $3 \mathrm{~cm}$ beyond the lower edge of the fistula. The patient was weaned from anesthesia in the operating room with the side arm of the T-tube occluded soon after surgery on the same day.

The patient recovered uneventfully and was discharged 7 days postoperation. The T-tube was replaced after a year via a rigid bronchoscope because of upward displacement and interference with the movement of the right vocal cord. The patient reported feeling comfortable with normal eating and speaking, and no residual fistula was detected during the subsequent 8-month follow-up period.

\section{Case 2}

A 26-year-old man with postintubation TEF and SLTS was admitted 4 months after emergency intubation and tracheostomy after a car accident. Bronchoscopy and computed tomography showed a circumferential stenosis at the cricoid level (5 $\mathrm{mm}$ at the narrowest diameter) and a giant TEF $(3.0 \mathrm{~cm}$ diameter) just below the stricture ring ( - Fig. 1A, B, C, D).

The patient had received long-term antibiotic and steroid therapies in other hospitals due to active psoriasis, ankylosing spondylitis, and left-lower-lobe pneumonia. Given the patient's poor condition, a draining gastrostomy and feeding jejunostomy were performed first. Then, 4 months later, the patient was readmitted, and the steroid therapy was discontinued for a month. Although the pneumonia was cured, radical airway reconstruction remained contraindicated due to the ankylosing spondylitis and poor skin condition from psoriasis. A conservative separation of the respiratory and alimentary tracts was performed, including fistula division, esophagus closure, muscle flap interposition, and tracheal membrane repair as in case 1 . Due to poor healing from the psoriasis, the tracheostomy was retained to facilitate respiratory drainage. Although the patient had some cough due to fluid intake at the time of discharge, no residual fistula was detected by a digestive endoscopy.

Six months later, a second tracheal T-tube treatment was performed. The preoperative bronchoscopy showed a 3-mm residual TEF buried in the granulation tissue below the cricoid plate. The scar tissue in the subglottic area and upper airway was decorticated. To provide more room to repair the fistula and expand the airway lumen, the anterior arch of the cricoid cartilage was resected subperichondrially. Two 4-0 PDS stitches were used to close the residual TEF from inside the tracheal lumen. A very thin layer of muscle flap was harvested to cover the repaired fistula from the inside and interpose the T-tube and sutures. A 13-mm Montgomery T-tube was implanted to support the airway. The patient recovered uneventfully and has had good quality of life during the subsequent 4-month follow-up period without evidence of fistula recurrence.

\section{Discussion}

Tracheal stenosis occurs in $70 \%$ of patients with postintubation TEF due to circumferential tracheal injury from cuff compression. ${ }^{1}$ SLTS is another postintubation complication, often due to circumferential cricoid erosion by the endotracheal tube or proximal erosion of the cricoid by a high or violent tracheostomy. Both patients in this report had a history of long-term intubation and tracheostomy. Pathologically, concurrent TEF and SLTS are usually due to two different etiologies: cuff compression and tracheostomy injury with long-segment or multisegment lesions predominating.

Laryngotracheal resection with primary reconstruction is the standard treatment for SLTS. ${ }^{3}$ However, there have been few reported cases of TEF complicated with SLTS. A study in a large group of patients with postintubation TEF showed that approximately $29 \%$ of patients could not be weaned from the tracheal appliances after surgical resection. These previous studies reported that the coexistence of glottic lesions was the primary cause of decannulation failure. $^{2,4}$ Additionally, a long-segment tracheal lesion $(>4 \mathrm{~cm})$ and giant fistula $(>3 \mathrm{~cm})$ are also predictors of resection failure.

We selected T-tube implantation in our two cases due to concurrent ankylosing spondylitis, recent steroid usage, and central nervous system damage. The Montgomery T-tube can correct subglottic airway stenosis effectively, and there is no evidence showing a negative effect of T-tube implantation on the healing of a newly repaired membrane. However, the importance of muscle flap interposition should be addressed, because wound contamination by respiratory secretion leaks is often inevitable around the T-tube. The muscle flap can protect the newly repaired trachea and esophagus. A recurrent or residual fistula is often more challenging than a primary lesion for surgical repair. The residual fistula in the second case was likely due to the patient's generally poor condition. Additionally, the fistula was just below the posterior cricoids. Therefore, the surgical exposure was very difficult. Currently, reisolating the trachea and esophagus is a serious challenge, because the surrounding tissue is likely to be injured. We successfully used a thin muscle flap to enhance the intralumen repair, though the long-term outcomes need to be further observed. Kalmár reported one case with similar technique for a primary repair of nonmalignant tracheogastric fistula following esophagectomy. ${ }^{5}$ T-tube displacement occurred in one case, which needed to be corrected because of the narrow window left by the subglottic space. However, the best replacement time is when the stoma is stable. To solve the diameter mismatch of the cricoid ring and distal airway, we wedge-cut the posterior wall of the proximal tip of the $T$ tube or removed the anterior arch of the cricoid cartilage subperichondrially with a very satisfactory outcome. 

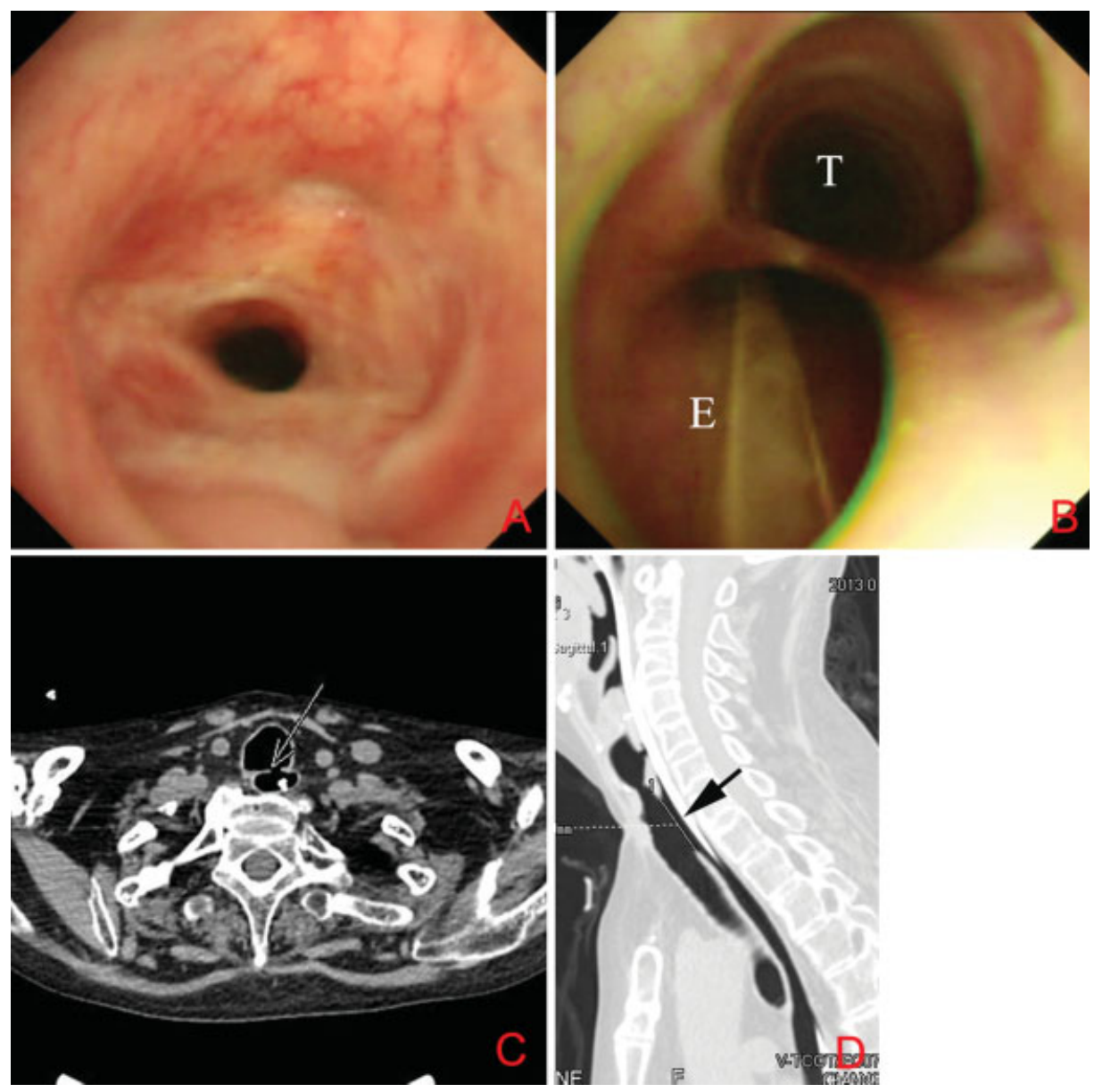

Fig. 1 Postintubation tracheoesophageal fistula (TEF) with synchronic subglottic laryngotracheal stenosis. (A) Circumferential stenosis at the cricoid level; (B) a giant TEF (diameter $3.0 \mathrm{~cm}$ ) just below the stricture ring; (C, D) fistula and airway stenosis (arrow) visualized on the computed tomography scan.

\section{Conclusion}

Postintubation TEF complicated with SLTS is a challenging surgical repair. In cases where radical surgical resection and reconstruction is contraindicated, T-tube implantation may prove to be a good alternative.

\section{References}

1 Camargo JJ, Machuca TN, Camargo SM, Lobato VF, Medina CR. Surgical treatment of benign tracheo-oesophageal fistulas with tracheal resection and oesophageal primary closure: is the muscle flap really necessary? Eur J Cardiothorac Surg 2010;37(3): 576-580

2 Muniappan A, Wain JC, Wright CD, et al. Surgical treatment of nonmalignant tracheoesophageal fistula: a thirty-five year experience. Ann Thorac Surg 2013;95(4):1141-1146

3 Mathisen DJ, Grillo HC, Wain JC, Hilgenberg AD. Management of acquired nonmalignant tracheoesophageal fistula. Ann Thorac Surg 1991;52(4):759-765

4 Macchiarini P, Verhoye JP, Chapelier A, Fadel E, Dartevelle P. Evaluation and outcome of different surgical techniques for postintubation tracheoesophageal fistulas. J Thorac Cardiovasc Surg 2000;119(2):268-276

5 Kalmár K, Molnár TF, Morgan A, Horváth OP. Non-malignant tracheo-gastric fistula following esophagectomy for cancer. Eur J Cardiothorac Surg 2000;18(3):363-365 